\title{
Extensive Hematoma in the Superficial Posterior Compartment with Threatening Compartment Syndrome after Ambulatory Phlebectomy
}

\author{
Wendy S. J. Malskat, MD, * and Marianne G. R. De Maeseneer, MD, PhD*†
}

The authors have indicated no significant interest with commercial supporters.

W e report a serious bleeding complication after ambulatory phlebectomy (AP) in a patient receiving anticoagulant treatment. AP is a surgical technique used to remove palpable and visible varicose veins. The Swiss physician Muller introduced it in its current form in 1956, using 2-mm incisions, no skin sutures or vein ligatures, local anesthesia, and immediate postoperative ambulatory compression. This procedure is frequently used in the treatment of superficial varicose veins. ${ }^{1-3}$ It is generally considered a safe procedure, with a low complication rate in experienced hands. ${ }^{1-4}$

\section{Case}

A 71-year-old man presented to our outpatient clinic with extensive varicose veins in both legs. Clinical history further revealed stroke, familial hypertrophic cardiomyopathy, atrial fibrillation, hypertension, and benign prostate hypertrophy. The man had been on long-term anticoagulation (acenocoumarol) with regular international normalized ratio (INR) monitoring at a local anticoagulation clinic. Clinical examination revealed extensive varicose veins in both legs with ankle edema and focal pigmentation (C2, C3, C4a).

Duplex ultrasound demonstrated incompetence of the great saphenous vein (GSV), with a large diameter at mid-thigh (right $8 \mathrm{~mm}$, left $11 \mathrm{~mm}$ ) and multiple incompetent tributaries. Treatment strategy consisted of endovenous thermal ablation of the main GSV trunk in two sessions, followed by AP sessions 2 months later, all performed in an office setting, without discontinuing anticoagulant treatment. No blood sample was taken to determine INR again immediately before AP, according to the policy at that time in our department (based on the fact that followup at an anticoagulation clinic is usually performed carefully in the Netherlands). Phlebectomies were performed under local anesthesia using lidocaine 1\% without adrenaline. Where considered appropriate, tributaries and perforating veins were ligated. Postoperative compression consisted of an elastic stocking with 25- to 30-mmHg ankle pressure.

Two days after the AP session at the medial side of the right leg, the patient presented urgently at the outpatient clinic with progressive severe pain and extensive swelling of the calf. The pain had started a few hours after the procedure. Clinical examination revealed a swollen right calf (diameter $7.5 \mathrm{~cm}$ greater than the left calf) with extensive bruising. The right pedal arteries were palpable and normal. Sensory and motor function of the leg and foot were intact, but the slightest active or passive movement of the ankle was extremely painful. Ultrasound examination showed a large hematoma in the superficial posterior compartment. There was no deep or superficial vein thrombosis.

\footnotetext{
*Department of Dermatology, Erasmus Medical Centre, Rotterdam, The Netherlands; ${ }^{\dagger}$ Department of Surgery, Faculty of Medicine and Health Sciences, University of Antwerp, Antwerp, Belgium
} 


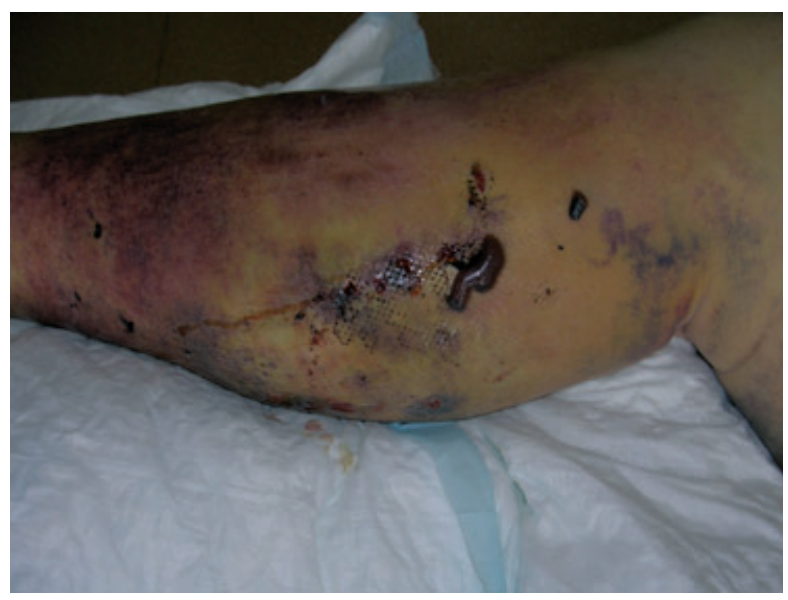

Figure 1. Extensive swelling, ecchymosis, and multiple blood blisters on the skin of the right calf 1 week after ambulatory phlebectomy. Initial conservative treatment with bed rest and elevation.

A threatening compartment syndrome was suspected, so the patient was immediately referred from our clinic to the emergency department. Laboratory testing confirmed an INR of 4.0. Repeat ultrasound examination revealed that the large hematoma was limited to one compartment, so it was initially decided to treat the patient conservatively with leg elevation and analgesics. Although the pain diminished slightly, swelling persisted, and the patient was not able to mobilize. During this time, perfusion of the skin over the hematoma became compromised, with several blood blisters appearing on the medial side of the right leg (Figure 1), so 2 days after admission, it was decided to proceed to surgical evacuation of the hematoma. A large hematoma was visible after opening the fascia (Figure 2), but the gastrocnemius muscle appeared to be viable. Because there was no active bleeding or muscular edema, the incision was closed, leaving a suction drain for 24 hours. Immediately after surgery, the patient's clinical condition improved dramatically, and he was able to mobilize a day later. There were no further skin problems or any neurologic impairment, and the surgical wound healed normally. At 2-month follow-up, recovery was complete, with no residual complications (Figure 3).

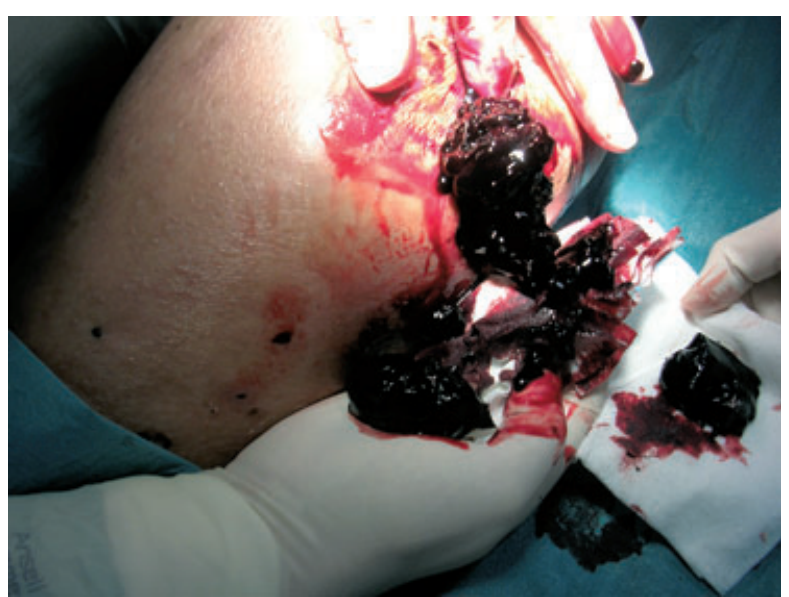

Figure 2. Evacuation of hematoma through a small medial fasciotomy.

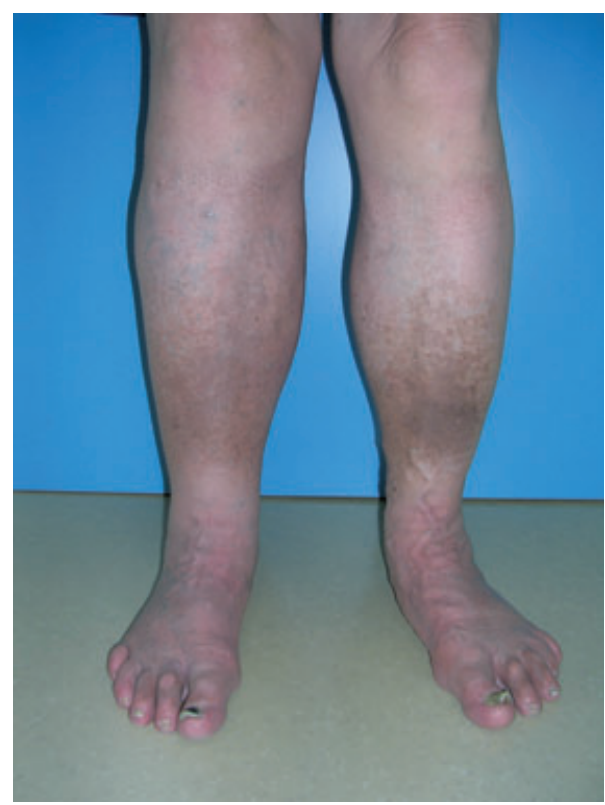

Figure 3. Normalized circumference and appearance of the right leg at 2-month follow-up.

\section{Discussion}

This is, to our knowledge, the first case report of an extensive hematoma in the superficial posterior compartment of the leg causing threatening compartment syndrome (CS) after AP. CS is a limbthreatening condition usually associated with traumatic injury, burns, crush or reperfusion injuries, or venous occlusion, resulting in a dramatic pressure 
increase within the muscular compartments due to edema or hematoma. Urgent fasciotomy is indicated to prevent muscle necrosis and subsequent loss of the limb in cases of acute CS.

The patient presented here developed a large intrafascial hematoma shortly after AP of the medial side of the leg. We hypothesize that a perforating vein was disrupted during AP, or a ligature slipped off during mobilization after the procedure. Because the patient was undergoing anticoagulant treatment, excessive bleeding occurred in the superficial posterior compartment, leading to the evolving clinical scenario described above. Although this compartment is much larger than the anterior or deep posterior compartments, a large hematoma may lead to a CS.

Caution is always warranted when planning surgical interventions in patients undergoing chronic anticoagulation, and for major surgery, INR should not usually exceed 1.5 on the day of surgery. There are no specific guidelines for target INR for patients taking anticoagulants undergoing AP. Because this is considered a minor intervention, INR monitoring is usually not mentioned, but based on the present experience, we suggest that INR should be monitored before AP if anticoagulation is to continue, in particular when large tributaries and perforating veins are involved.

In general, bleeding complications of AP are rare. In a review article, Ramelet ${ }^{1}$ reported postoperative hemorrhages in $0.3-4.3 \%$ of cases, whereas major hematomas were observed in $0.1-2.4 \%$. If it is decided to perform AP in a patient undergoing anticoagulant treatment, it should be performed carefully. Instead of simple avulsion, ligation of perforating veins and tributaries directly connected to the "source of reflux" is recommended, which requires preoperative marking with duplex ultrasound. ${ }^{4}$ In addition, the use of local anesthetic with adrenaline or even tumescent anesthesia may reduce bleeding. ${ }^{5}$ Careful application of postoperative dressing and compressive bandaging (or stocking) and a short period of postoperative observation of the patient (10-30 minutes) are mandatory measures to reduce the incidence of, or recognize quickly, bleeding complications. $^{2,3}$ Some authors recommend seeing the patient for a first follow-up visit routinely 1 or 2 days after the operation., ${ }^{2,3}$ All of these measures may be useful in patients at high risk of bleeding, as presented here.

AP is usually painless or only moderately uncomfortable. The presented case illustrates that severe or increasing pain after the procedure must always be considered as a cause of serious concern and should be urgently investigated.

\section{References}

1. Ramelet AA. Phlebectomy. Technique, indications and complications. Int Angiol 2002;21:46-51.

2. Kabnick LS, Ombrellino M. Ambulatory phlebectomy. Semin Intervent Radiol 2005;22:218-24.

3. Almeida JI, Raines JK. Ambulatory phlebectomy in the office. Perspect Vasc Surg Endovasc Ther 2008;20:348-55.

4. Olivencia JA. Pitfalls in ambulatory phlebectomy. Dermatol Surg 1999;25:722-5.

5. Keel D, Goldman MP. Tumescent anesthesia in ambulatory phlebectomy: use of epinephrine. Dermatol Surg 1999;25:371-2.

Address correspondence and reprint requests to: Marianne De Maeseneer, Department of Dermatology, Erasmus Medical Centre, PB 2040, 3000 CA Rotterdam, The Netherlands, or e-mail: m.demaeseneer@erasmusmc.nl 